\title{
Dijets in Diffractive Photoproduction and Diffractive Factorisation
}

\author{
Paul Newman (for the H1 Collaboration) \\ School of Physics \& Astronomy, University of Birmingham, B15 2TT, UK
}

\begin{abstract}
This contribution [1] reports on a recent measurement of diffractive dijet photoproduction, which is used to study the dynamics of absorptive (multiple scattering) effects associated with the presence of beam remnants in diffractive hard scattering processes. The corresponding rapidity gap survival probability is extracted as a function of several variables from comparisons between data and NLO QCD calculations and is found to be roughly constant at about 0.5 . There is no evidence for the expected dependence on the momentum fraction of the photon entering the hard scattering, $x_{\gamma}$. The ratio of the diffractive to the inclusive dijet photoproduction cross sections is also studied.
\end{abstract}

\section{Introduction}

Over the past 15 years, measurements by the HERA experiments have led to a revolution in our understanding of diffractive scattering at the microscopic level in terms of QCD and partonic interactions. Inclusive diffractive deep-inelastic scattering (DDIS) events of the type $e p \rightarrow e X p[2,3]$, have been used to extract Diffractive Parton Distribution Functions (DPDFs) $[2,4,5,6]$ in the framework of a semi-inclusive hard scattering collinear factorisation theorem [7]. The DPDFs have been found to be dominated by gluons and to good approximation exhibit a 'proton vertex factorisation' property, whereby they vary only in normalisation as the four-momentum of the final state proton changes.

Given a knowledge of the DPDFs, NLO perturbative QCD calculations have successfully predicted other DDIS observables at HERA, including dijet [5, 8], heavy quark [9] and longitudinal photon-induced [10] cross sections. However, as has long been expected [7, 11], DPDF-based predictions for hard diffractive processes in $p \bar{p}$ scattering fail by around an order of magnitude to describe the data [12]. This factorisation breaking is generally attributed to absorptive corrections, corresponding to the destruction of the outgoing proton coherence and the rapidity gap due to multiple interactions within a single event. These effects are associated with the presence of a proton remnant in the event, in contrast to the point-like photon coupling in the DDIS case. The corresponding 'rapidity gap survival probability' can be treated semi-quantitatively [13] and its prediction at LHC energies is a major current issue [14]. It is therefore essential to test models of absorptive effects in as many applications as possible.

The issues of DPDF applicability and rapidity gap survival can be studied at HERA in hard diffractive photoproduction, where the virtuality of the exchange photon coupling to the electron is close to zero. Under these circumstances, the photon can develop an effective partonic structure via $\gamma \rightarrow q \bar{q}$ fluctuations and further subsequent splittings. In a simple leading order picture, there are thus two classes of hard photoproduction as illustrated in figure 1: 'resolved' interactions, where the photon interacts via its partonic structure and only a fraction $x_{\gamma}$ of its four-momentum participates in the hard subprocess and 'direct' interactions, where the photon enters the hard interaction as a point-like particle and $x_{\gamma}=$ 1. Resolved photoproduction interactions resemble hadron-hadron scattering to a large 
extent [15] and are therefore expected to exhibit gap destruction effects. The gap survival probability has been estimated in a phenomenological model to be 0.34 for resolved processes [16] and is expected to be unity for direct photon interactions.

Previous $\mathrm{H} 1$ analyses of diffractive dijet photoproduction [17] have consistently measured cross sections to be smaller than theoretical predictions, suggesting rapidity gap survival probabilities of around 0.5. A ZEUS measurement at somewhat larger jet transverse energies $\left(E_{T}^{\text {jet }}\right)[6,18]$ yielded a larger survival probability, compatible with unity. Neither collaboration has found any evidence for the expected $x_{\gamma}$ dependence and both have found a suggestion of a stronger $E_{T}^{\text {jet }}$ dependence in the data than is predicted by NLO theory [19], which may explain the apparent difference between the $\mathrm{H} 1$ and ZEUS findings.
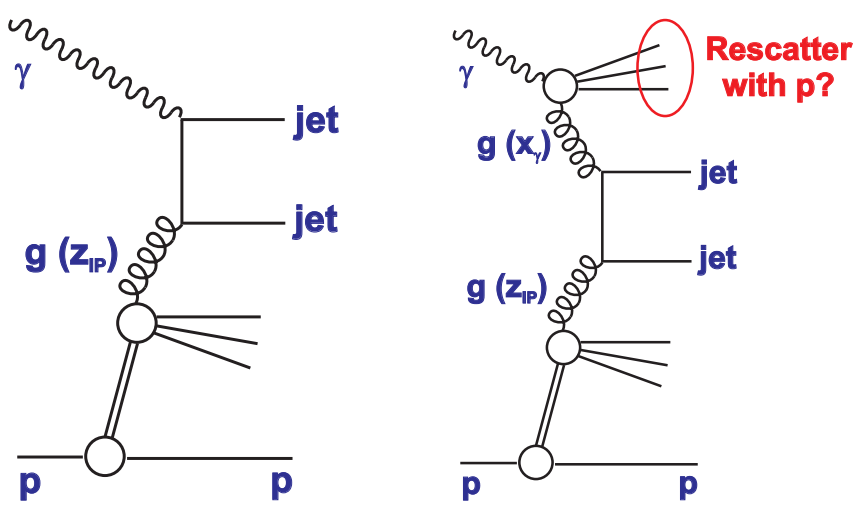

Figure 1: Example leading order diagrams corresponding to diffractive dijet photoproduction initiated by direct (left) and resolved (right) photons. The momentum fractions $x_{\gamma}$ of the photon and $z_{\mathbb{P}}$ of the diffractive exchange are indicated.

At present there is no completely satisfactory theoretical interpretation of these observations.

\section{Data and Experimental Method}

New diffractive dijet photoproduction cross section measurements have been made by the H1 collaboration using a sample with an integrated luminosity of $47 \mathrm{pb}^{-1}$, about a factor three larger than previously studied. After obtaining a sample of diffractive events using the standard H1 Large Rapidity Gap selection [2], the hadronic final state is subjected to the $k_{T}$ longitudinally invariant jet algorithm [20], applied in the laboratory frame with parameters $R=1$ and $p_{T}^{\text {jet,min }}=2.5 \mathrm{GeV}$. The cross sections are measured in the kinematic range:

$$
\begin{aligned}
& Q^{2}<0.01 \mathrm{GeV}^{2} \quad 0.3<y<0.65 \\
& E_{T}^{\text {jet1 }}>5 \mathrm{GeV} \quad E_{T}^{\text {jet } 2}>4 \mathrm{GeV} \\
& -1<\eta^{\text {jet1 }}<2 \quad-1<\eta^{\text {jet } 2}<2 \\
& x_{\mathbb{P}}<0.03 \quad M_{Y}<1.6 \mathrm{GeV} \quad|t|<\mathrm{GeV}^{2} .
\end{aligned}
$$

Here, the variables $Q^{2}$ and $y$ have their conventional DIS meaning, $E_{T}^{\text {jet } 1}$ and $E_{T}^{\text {jet2 }}$ are the transverse energies of the leading and next-to-leading jets, $\eta^{\text {jet } 1}$ and $\eta^{\text {jet2 }}$ are the corresponding jet pseudorapidities in the laboratory frame, $x_{\mathbb{P}}$ is the fractional momentum of the incoming proton which is transferred to the diffractive exchange, $M_{Y}$ is the mass of the outgoing baryonic system (dominantly a proton) and $t$ is the squared four-momentum transfer to the proton. 
The data are compared with NLO calculations obtained from [21] with the H1 2006 Fit A and B [2] and H1 2007 Fit Jets [5] DPDFs. The resolved photon is described with the GRV HO photon parton densities [22]. The DIS- $\gamma$ scheme is used with four quark flavours, $\Lambda_{4}=330 \mathrm{MeV}$ and the factorisation and renormalisation scales set to $E_{T}^{j e t 1}$. Hadronisation corrections are applied to the parton level calculations using the RAPGAP Monte Carlo model [23].

\section{Diffractive Cross Sections and NLO QCD Calculations}

Figure 2 shows the ratio of the measured diffractive dijet photoproduction cross section to the NLO QCD calculation differentially in $E_{T}^{j e t 1}$ and in hadron level estimators of $z_{\mathbb{P}}$ and $x_{\gamma}$, obtained as described in [17]. For most of the measured points, the ratios are significantly below unity. When taking the H1 Fit B DPDFs, which describe a wide range of DDIS observables, there is little dependence of the ratio on $z_{\mathbb{P}}$. The other two DPDF sets give ratios which are consistent with $\mathrm{H} 1 \mathrm{Fit} \mathrm{B}$ at low $z_{\mathbb{P}}$, but deviate at large $z_{\mathbb{P}}$, where inclusive diffractive data give poor constraints on the gluon density [2].

Integrated over the full measured range, the ratio $S$ of data to theory using Fit B is

$$
\begin{aligned}
& S=0.54 \pm 0.01 \text { (stat) } \pm 0.10 \text { (syst) } \\
& \pm 0.13 \text { (scale) },
\end{aligned}
$$

where the first two uncertainties are experimental and the third is the result of varying the factorisation and renormalisation scales in the theory by factors of 0.5 and 2 . The results when using the $\mathrm{H} 1$ Fit $\mathrm{A}$ and $\mathrm{H} 1$ Fit Jets DPDFs are $S=0.43$ and $S=0.65$ respectively, with similar uncertainties to the Fit B case. Including the differences between the different DPDF sets in the uncertainties and integrating over the full measured range, the rapidity gap survival probability based on Fit B is smaller than unity by
H1 PRELIMINARY
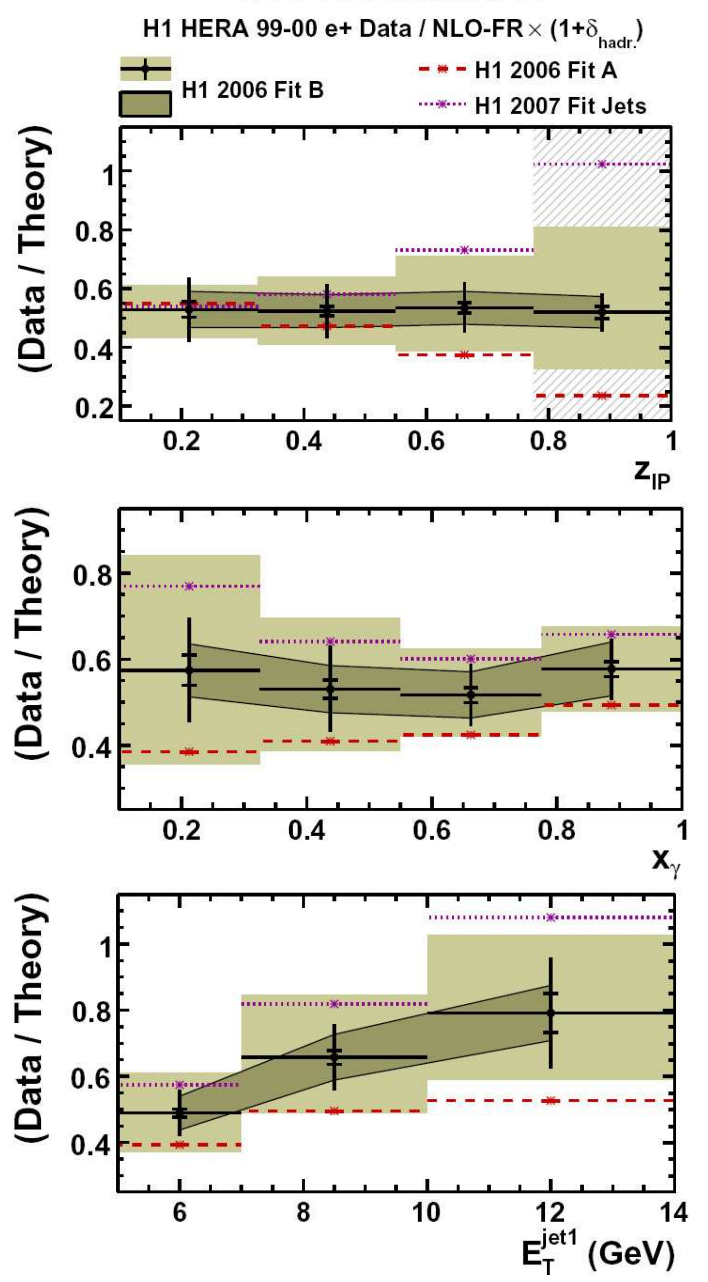

Figure 2: Ratios of measured cross sections to NLO QCD calculations as a function of $z_{\mathbb{P}}$, $x_{\gamma}$ and $E_{T}^{j e t 1}$. The inner error bars on the data points are statistical and the outer error bars show the quadratic sum of the statistical and uncorrelated systematic errors. The dark band shows the experimental systematics which are correlated between the data points. The light band shows the scale uncertainty on the NLO theory. The results obtained when replacing the H1 2006 DPDF Fit B partons with two other DPDF sets are also shown. 
$2.4 \sigma$, confirming [17].

Notably, the measured ratio has only a weak dependence on $x_{\gamma}$, in contrast to theoretical expectations $[16,24]$. On the other hand, some evidence for a dependence of the ratio on $E_{T}^{j e t 1}$ is observed. Since the correlations between the variables are complicated (e.g. $E_{T}^{j e t 1}$ and $x_{\gamma}$ are strongly positively correlated through the kinematic restrictions), more differential studies are required to fully unfold the dynamics.

\section{Diffractive to Inclusive Cross Section Ratios}

Measurements of ratios of diffractive to inclusive dijet photoproduction cross sections have been proposed as a further test of the dynamics of gap survival issues [16], and have been studied further via NLO calculations in [24]. Their potential advantages over straight-forward diffractive measurements lie in the partial cancellations of some experimental systematics as well as theoretical uncertainties arising from the photon structure and factorisation and renormalisation scale choices. A similar ratio was measured by the CDF collaboration [12] as a means of extracting effective $p \bar{p}$ DPDFs for comparison with HERA predictions and assessment of gap survival probabilities.

In the low transverse momentum regime, underlying event or multi-parton interactions are known to have a non-negligible influence on inclusive photoproduction jet cross sections [25]. Here, the underlying event is modelled using the PYTHIA MC generator, which produces multiple partonparton interactions in the same event, tunable largely via a parameter $p_{\perp \text { min }}$, corresponding to the minimum transverse momentum down to which secondary scattering is calculated.

Here, the inclusive photoproduction cross section is measured, using data from the same running period as in the diffractive case, in a kinematic range defined by the $Q^{2}$, $y$ and jet $E_{T}$ and $\eta$ ranges of the diffractive measurement. The ratio of diffractive to inclusive cross sections is formed as a function of several variables and shown for the example case of $x_{\gamma}$ in figure 3 . The data are compared with the predicted ratios based on the RAPGAP and PYTHIA Monte Carlo models, both before and after underlying event effects

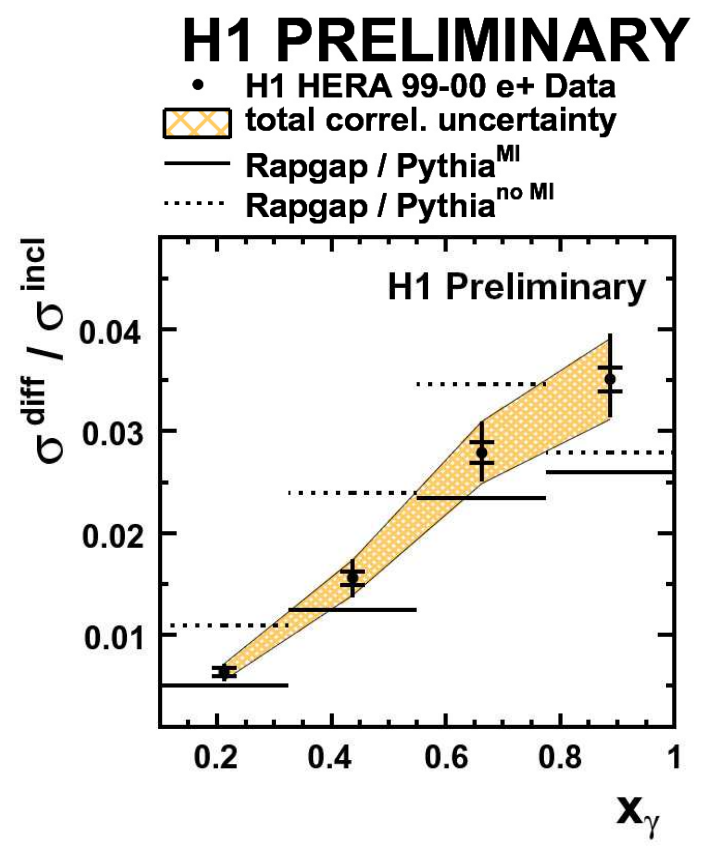

Figure 3: Ratio of the diffractive to the inclusive dijet photoproduction cross section as a function of $x_{\gamma}$. The inner error bars correspond to statistical uncertainties. The outer error bars contain in addition the uncorrelated systematic uncertainties. Correlated systematic errors are indicated by the band. The data are compared with predictions formed from the ratio of the RAPGAP to the PYTHIA Monte Carlo model, both with and without an underlying event simulation in the inclusive (PYTHIA) case.

DIS 2009 
are taken into account in PYTHIA. The PYTHIA underlying event corrections change the predicted inclusive cross sections, and hence the ratios, by up to a factor of two at low $x_{\gamma}$, diminishing as $x_{\gamma}$ increases. This effect is similar in size to the gap survival probabilities suggested by the diffractive data in section 3 . The large, but unquantified, uncertainties in modelling the underlying event therefore preclude strong conclusions on the basis of the ratio data at this stage. Nonetheless, the RAPGAP / PYTHIA ratio, with multiple interactions included, describes the data rather well.

\section{References}

[1] Slides: http://indico.cern.ch/contributionDisplay $\cdot$ py?contribId=124\&sessionId=18\&conf Id=53294

[2] H1 Collaboration, Eur. Phys. J. C 48 (2006) 715 [hep-ex/0606004].

[3] ZEUS Collaboration, Nucl. Phys. B 816 (2009) 1 [hep-ex/0812.2003]; M. Ruspa, M. Kapishin, these proceedings.

[4] A. Martin, M. Ryskin and G. Watt, Eur. Phys. J. C 44 (2005) 69 [hep-ph/0504132].

[5] H1 Collaboration, JHEP 0710 (2007) 042 [hep-ex/0708.3217].

[6] W. Slominski, these proceedings.

[7] J. Collins, Phys. Rev. D 57 (1998) 3051, erratum ibid. D 61 (2000) 019902 [hep-ph/9709499].

[8] ZEUS Collaboration, Eur. Phys. J. C 52 (2007) 813 [hep-ex/0708.1415].

[9] H1 Collaboration, Eur. Phys. J. C 50 (2007) 1 [hep-ex/0610076]; ZEUS Collaboration, Nucl. Phys. B 672 (2003) 3 [hep-ex/0307068].

[10] D. Salek, these proceedings.

[11] J. Collins, L. Frankfurt and M. Strikman, Phys. Lett. B 307 (1993) 161 [hep-ph/9212212]; A. Berera and D. Soper, Phys. Rev. D 50 (1994) 4328 [hep-ph/9403276].

[12] CDF Collaboration, Phys. Rev. Lett. 84 (2000) 5043.

[13] A. Kaidalov, V. Khoze, A. Martin and M. Ryskin, Eur. Phys. J. C 21 (2001) 521 [hep-ph/0105145].

[14] M. Arneodo, M. Diehl, V. Khoze and P. Newman, 'Working Group on Diffraction: Executive Summary' and references therein, proceedings of the workshop: HERA and the LHC, 2006-8 [hep-ph/0903.3861].

[15] J. Butterworth and M. Wing, Rept. Prog. Phys. 68 (2005) 2773 [hep-ex/0509018].

[16] A. Kaidalov, V. Khoze, A. Martin and M. Ryskin, Phys. Lett. B 567 (2003) 61 [hep-ph/0306134].

[17] H1 Collaboration, Eur. Phys. J. C 51 (2007) 549 [hep-ex/0703022].

[18] ZEUS Collaboration, Eur. Phys. J. C 55 (2008) 177 [hep-ex/0710.1498].

[19] K. Cerny, 'Diffractive Photoproduction of Dijets in ep Collisions at HERA', proceedings of DIS08 (London), April 2008.

[20] S. Catani, Yu. Dokshitzer, B. Webber, Phys. Lett. B 285 (1992) 291.

[21] S. Frixione, Z. Kunzst and A. Signer, Nucl. Phys. B 467 (1996) 399 [hep-ph/9512328];

S. Frixione, Nucl. Phys. B 507 (1997) 295 [hep-ph/9706545];

M. Klasen and G. Kramer, Eur. Phys. J. C 38 (2004) 93 [hep-ph/0408203].

[22] M. Glück, E. Reya and A. Vogt, Phys. Rev. D 45 (1992) 3986; M. Glück, E. Reya and A. Vogt, Phys. Rev. D 46 (1992) 1973.

[23] H. Jung, Comput. Phys. Commun. 86 (1995) 147.

[24] M. Klasen and G. Kramer, Mod. Phys. Lett. A 23 (2008) 1885 [hep-ph/0806.2269].

[25] H1 Collaboration, Z. Phys. C 70 (1996) 17 [hep-ex/9511012];

H1 Collaboration, Eur. Phys. J. C 29 (2003) 497 [hep-ex/0302034]. 